\title{
Low Carbon City and Analysis of its Guidance to Urban Planning
}

\author{
Jun $\mathrm{Ma}^{1, \mathrm{a}}$, Shengxiao $\mathrm{Li}^{1, \mathrm{~b}}$ and Chaorong Zhang ${ }^{2, \mathrm{c}}$ \\ ${ }^{1}$ Architectural Design and Research Institute, Shenyang Jianzhu University, Shenyang, 110168, \\ China P.R. \\ ${ }^{2}$ College of Civil Engineering, Shenyang Jianzhu University, Shenyang, 110168,China P.R. \\ wwasyn@163.com , ${ }^{\mathrm{a}}$ lishengxiao@163.com, ${ }^{\mathrm{c} 603045063 @ q q . c o m}$
}

\section{Keywords: Low Carbon City; Urban Planning; Guidance}

Abstract. In this paper, we discuss how to combine the idea of sustainable development with urban planning as a public policy, and support the construction of low carbon cities by scientific and reasonable planning according to the concept of low carbon city. The main points of the paper are the guidance and the demands of the construction of low carbon cities to urban planning consisting of spatial planning,transportation planning and industry planning.

\section{Introduction}

Since the industrial revolution, science and technology to the human society into a new era of mobile, mass production and high density of population structure of global resources and the ecological landscape, significant changes have taken place, people gradually realize the industrialization in the future the threat of human survival environment, rethinking the industrial development pattern. In December 1997 held in Kyoto, Japan, the United Nations framework convention on climate change by third party meeting, representatives of 149 countries and regions by the designed to limit greenhouse gas emissions in the developed countries to curb global warming, the Kyoto protocol, formally went into effect on February 16, 2005. In 2003 promulgated the "energy white paper" for low-carbon development model proposes the detailed goals and a road map. Since then, countries around the world continue to low carbon development on the national important strategy, also agreed to share responsibilities for energy conservation and emissions reduction. China since May 1998 signed and approved in August 2002 since the Kyoto protocol, also began to actively explore and research the low carbon economy mode; Held in Copenhagen in 2009 the United Nations framework convention on climate change of the 15th on the conference of the parties, the Chinese government promised in 2005, on the basis of the base period, per unit of GDP carbon emissions by $40 \%$ by $2020-45 \%$. And cities as the important carrier of industrial civilization, due to its gathered a large number of population and the production and living activities, carbon emissions accounted for $75 \%$ of global carbon emissions, how to planning, development, construction low carbon cities become realize low carbon development, should be the key to global climate change. carbon emission calculation of thermal power plant - an overview; impact of climate change on regional economy; design of carbon efficient online logistics networks; study on influencing factors of China's carbon emission rights price; study on the multi-target system of building energy efficiency engineering in China; a short review of wastewater biological treatment at low $\mathrm{C} / \mathrm{N}$ ratio; the construction of support system of China's government green procurement; carbon footprint accounting practices in Taiwan; research on EMC promote green commercial buildings form; simulation analysis of a printing factory energy saving; food packaging design exploration with the concept of low carbon; study on model of carbon emission rights allocation 
among power enterprises; effects of energy prices on energy efficiency in China; the risk analysis of new energy power generation; problem of China's nuclear security supervision system; energy consumption analysis of ship energy system; renewable energy evaluation and plan in Matsu areas; study on heat and power technology of heavy oilfield; short-term power load forecast in electric companies; energy management of industrial installation; the definition and identification of natural resources industries; China's sustainable development, challenges and solutions

\section{The Concept Of Low Carbon City}

Since the low carbon concept,scholars for the study of the theory of the low carbon economy are more enthusiastic, and low carbon cities is still in the early research, relatively both in theory and concept to promote practice at the exploration stage. In recent years, people gradually realized that to control carbon emissions, realize the energy conservation and emissions reduction, should first focus on urban areas with large population, industry, especially the big cities of a certain scale, here is the "crystallization" of modern industrial civilization, and people all kinds of energy production and living activities, the important source of greenhouse gases, as a result, more and more cities in the world began to explore establishing model for the development of low carbon city, our country also has more and more researchers put forward its own views on low carbon city.Kunpu Xia think low-carbon city is a low carbon economy in the city, including low-carbon production and low-carbon consumption, and build a resource-conserving and environment-friendly society, build a benign ecological sustainable energy system.Zhilin Liu and Yixin Dai scholars think such as low carbon city shall be understood as the mode of economic development, consumption idea and change the way of life, under the premise of guarantee the quality of life constantly improve, implementation help reduce carbon emissions way mode of urban construction and social development.Low-carbon city is a low carbon economy in the city, including low-carbon production and low-carbon consumption, and build a resource-conserving and environment-friendly society, build a benign ecological sustainable energy system. Low carbon city shall be understood as the mode of economic development, consumption idea and change the way of life, under the premise of guarantee the quality of life constantly improve, implementation help reduce carbon emissions way mode of urban construction and social development. Low-carbon city is a comprehensive system, to planning, construction and promote the policy and system; Low carbon city is the interaction between production and consumption, economic growth pattern and the way of urban development is the key; Low-carbon city is a kind of form, urban circular economy with low energy consumption, low pollution, low emission, high energy, high benefit, high cycle as the main characteristics. Low carbon city concept covers the visible energy technology, mode of production and life.In low carbon idea occupies a strategic position today, the role of governments at all levels should also attach great importance to planning, give full play to its policy guidance, to the guideline of sustainable planning, the construction of low carbon city. Construction of low-carbon city is a complex work, involving all aspects of policy requirements, the author here will give the public policy is roughly divided into city planning in spatial planning, traffic planning, industry planning, put forward from different angles for low-carbon city construction planning requirements. 


\section{The Urban Space Planning And Analysis Of The Low Carbon Development Guide}

In the urban construction, urban spatial structure for the city's efficiency has a direct guidance and restriction. A goal to fit the development of urban geography and reasonable urban spatial structure to guide the rational growth in cities, can lead to the efficient and orderly, the city's transportation can make urban life more convenient. Without reasonable urban spatial structure will become the obstacles for the sustainable development of city. Therefore, in order to match the requirement of low carbon city construction, government and the planners should make planning tend to be more scientific and reasonable. When making land use planning, pay attention to the proportion of various types of land equalization, every part of the development of need to concentrate in the city should increase the mixing degree of land function, in addition, still should pay attention to the plot of the scale of the various types of land, urban residents can use low carbon travel solutions to the diverse needs of life, work and leisure.

\section{Low Carbon Development To Guide Analysis Of Urban Traffic Planning}

Of the road traffic system planning in urban planning mainly includes for each city, the function arrangement and layout of road of main road cross section in the form of design. City road network is the basic skeleton of urban development system, determine the urban space form, also affect the plot of the land use planning of it and the urban development and construction direction has the close relationship and dweller, at the same time transportation of carbon emissions in the city's overall carbon emissions contributed at the forefront of the reasonable effective urban road system planning can promote urban efficiency, convenient life, also can promote urban energy conservation and emissions reduction. The current running status of most of the city road system in our country there are many problems, the most visual performance is traffic congestion, and find the reason from the planning is the level of road network.Structural imbalance and a decline in road network density. Now many of the road system planning is planning more than practicality, planning and road network expressway and distributor roads is too high, the proportion of the primary and secondary branch is far from enough, lack of sexual life road. Should vigorously develop public transportation, per carbon emissions are the lowest transportation public trolley. In the field of carbon concentration of transportation, adjust the structure of transportation is an effective way of energy conservation and emissions reduction, the government needs to vigorously develop public transport, including public trolley.The improvement of the line system, improve to the establishment of urban low-carbon transport system bring be reckoned. Encourage bicycles and walking. Compared with motorized transportation, bicycle and walk way with freedom, economic, energy saving, environmental protection, health, and many other advantages, human is becoming more and more under the background of advocating green lifestyle, bike and walk way to travel will be conducive to optimize traffic junction.Structure, reduce energy consumption and emissions.

\section{Urban Industrial Planning Of Low-carbon Development To Guide Analysis}

Urban industry structure with low carbon as the goal, both two aspects to transform traditional industries and the development of new industry, realize the optimization and upgrading of industrial structure. Realize the traditional industry to the industry diversification, clean production, utilization 
efficiency of the new industrial economic transformation, to maximize overall comprehensive benefit of economy and environment. From the perspective of overall regional coordinated development, overall planning, comprehensive consideration, the construction of regional low carbon economy industrial chain., on the basis of low carbon economy industrial chain in the city, in combination with the practical situation of city land layout and spatial structure, the construction of different low carbon industry economic zone.

\section{Conclusion}

Through research, the conclusions are as following:

(1) planning according to the urban development condition and target to determine the reasonable structure of the urban space, avoid group that concentrated outside the city constantly expanding or lack of group city group development, learning to explore TOD mode, looking for more efficient compact urban spatial structure.

(2) For more practical urban road network planning, reduce unnecessary road, increase of the city and the main living areas of the branch, improve the density of road network, and combining with the urban development goals accurately forecast the traffic demand, road section is determined.

(3) Overall regional coordinated development, to build a low-carbon economy industrial chain, promote the construction of low carbon industrial park.

(4) Optimize the structure of industry of city, to transform traditional industries and the development of new industries, and give attention to two or more things traditional industry diversification, clean and efficient production, at the same time, encourage the development of new type low carbon green industry.

(5) Suggested that the government support in the form of corresponding traffic policy low-carbon transport system, vigorously develop public transportation, speed up the construction of rail transit, control the quantity of private car travel, encourage bike travel and walking to ensure a variety of smooth transportation.

\section{References}

[1] Bent Flyvbjerg. Rationality and Power[M]. Chicago: The University of Chicago Press, 1998.

[2]Mill R, D Lessard. The Strategic Management of Large Engineering Projects: Shaping Risks, Institutions and governance[M]. Cambridge, MA: MIT Press, 2001.

[3]A $1 \mathrm{t}$ sh u 1 e r A 1 a n, D a vid L u be r off. M e g a - Proje c t s. The Changing Politics of Urban Public Investment[M].Washington, DC: Brooking Instituting Press.2003.

[4]Dennis Pamlin, Suzanne Pahlman,Elaine Weidman. A Five-step-planfor a Low Carbon Urban Development[Z]. 2009. 